\title{
ENTREVISTA
}

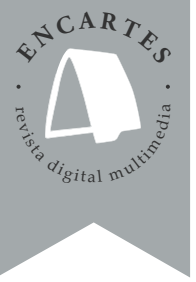

\section{MASGULINIDADES EN EL NORTE DE MÉXICO}

MASGULINITIES IN NORTHERN MEXICO

\author{
Entrevista de Joel Pedraza Mandujano* \\ Con Misael Hernández
}

Liga del video:

https://youtu.be/HxNEVOzrfHQ

Zl Dr. Misael Hernández, profesor investigador de El Colegio de la UFrontera con sede en Matamoros, habla en torno a cómo se construyen las nuevas masculinidades desde los estudios de género y los estudios culturales. El investigador hace un repaso histórico acerca del tema y la manera en que ha interactuado con otros campos, como los estudios queer. Profundiza en la manera en que el concepto de masculinidad hegemónica ha cambiado, tanto temporal como geográficamente, a lo largo de la historia desde la década de los ochenta hasta ahora.

$\mathrm{El}$ autor hace un comparativo entre el modo en que se construyen las masculinidades en distintos contextos geográficos e invita a reflexionar en torno a este concepto no como una idea sino como un proceso, como parte de un cambio continuo y moldeable. Acota los análisis sobre las masculinidades en el contexto fronterizo al hablar de "geopolíticas de la masculinidad", es decir, que conceptos como "masculinidad hegemónica" o "dominación masculina" tienen influencia estadounidense, australiana o francesa, por lo que construir desde adentro es imperativo y necesario para estructurar un concepto de masculinidad a partir de la condición fronteriza, basado en las jerarquías sociales.

\footnotetext{
* CIESAS-Occidente.

** Universidad Autónoma de Tamaulipas y El Colegio de Michoacán.
}

ISSN en trámite, Bajo licencia Creative Commons

Encartes 3 • marzo-agosto 2019, pp. 226-227

Recepción: 14 de febrero 2018 • Aceptación: 31 de mayo 2018 http://www.encartesantropologicos.mx

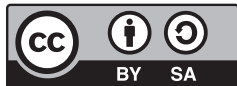


Foel Pedraza Mandujano es doctor en Ciencias Sociales por el CIEsas-Occidente. Sus temas de investigación abarcan migración, comunicación, tecnologías y juventud. Actualmente, es profesor de tiempo completo en la Universidad Intercultural del Estado de México. Entre sus publicaciones destacadas están: "Social networks and communicative meaning in Mexican Migration Networks in the US" en The digital transformation in public sphere (2016). Ed. Palgrave.

Misael Hernández es sociólogo, egresado de la Universidad Autónoma de Tamaulipas, y antropólogo social por El Colegio de Michoacán. Fue coordinador del libro: Masculinidades en el México comtemporáneo (2011), y es autor de los libros Masculinidades en Tamaulipas: Una historia antropológica (2012) e Historia, cultura y masculinidades en Tamaulipas (2013). Actualmente, realiza estudios sobre jóvenes, migración y masculinidad en la frontera Tamaulipas-Texas, así como en la frontera de Chiapas con Guatemala. 
Ángela Renée de la Torre Castellanos

Directora de Encartes

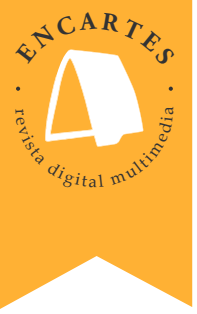

Arthur Temporal Ventura

Editor

Coordinación de Publicaciones del ciesas

Corrección, diseño y formación

Saúl Justino Prieto Mendoza

Difusión

CENTRO DE INVESTIGACIONES

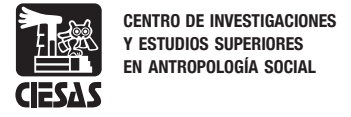

Encartes cuenta con el apoyo de El Colegio de la Frontera Norte y el Instituto Tecnológico y de Estudios Superiores de Occidente.

Equipo de coordinación editorial

Renée de la Torre Castellanos Directora de Encartes - Rodrigo de la Mora Pérez Arce iteso - Arcelia Paz CIESAS-Occidente - Santiago Bastos Amigo ciesas-Occidente Manuela Camus Bergareche Universidad de Guadalajara - Luis Escala Rabadán El COLEF

\section{Comité editorial}

Agustín Escobar Latapí Director general de ciesas - Alberto Hernández Hernández Presidente de El coLEF - Enrique Páez Agraz Director del Departamento de Estudios Socioculturales del iteso - Andrés Fábregas Puig ciesas-Occidente - Dulce Mariana Gómez Salinas Subdirectora del departamento de publicaciones de ciesas - Érika Moreno Páez Coordinadora del departamento de publicaciones de El COLEF - Manuel Verduzco Espinoza Director de la Oficina de Publicaciones del iteso - José Manuel Valenzuela Arce El colef - Luz María Mohar Betancourt ciesas-Ciudad de México - Ricardo Pérez Monfort CIESAS-Ciudad de México - Sévérine Durin Popy Ciesas-Noreste - Carlos Yuri Flores Arenales Universidad Autónoma del Estado de Morelos - Sarah Corona Berkin DEcs/Universidad de Guadalajara Norma Iglesias Prieto San Diego State University - Camilo Contreras Delgado El colef • Alejandra Navarro Smith ITESO

Cuerpo académico asesor

Alejandro Frigerio

Universidad Católica

Argentina-Buenos Aires

Alejandro Grimson

USAM-Buenos Aires

Alexandrine Boudreault-Fournier

University of Victoria-Victoria

Carlo A. Cubero

Tallinn University-Tallin

Carlo Fausto

UFRJ-Rio de Janeiro

Carmen Guarini

UBA-Buenos Aires

Caroline Perré

Centro de Estudios Mexicanos y

Centroamericanos-Ciudad de

México

Clarice Ehlers Peixoto

UERJ-Rio de Janeiro
Claudio Lomnitz

Columbia-Nueva York

Cornelia Eckert

Ufrgs-Porto Alegre

Cristina Puga

unam-Ciudad de México

Elisenda Ardèvol

Universidad Abierta de

Cataluña-Barcelona

Gastón Carreño

Universidad de

Chile-Santiago

Gisela Canepá

Pontificia Universidad

Católica del Perú- Lima

Hugo José Suárez

unAm-Ciudad de México

Jesús Martín Barbero

Universidad Javeriana-Bogotá

\section{Julia Tuñón}

INAH-Ciudad de México

María de Lourdes Beldi

de Alcantara

UsP-Sao Paulo

Mary Louise Pratt

NYU-Nueva York

Pablo Federico Semán

CONICET/unsam-Buenos Aires

Renato Rosaldo

NYU-Nueva York

Rose Satiko Gitirana Hikji

UsP-Sao Paulo

Rossana Reguillo Cruz

ITESO-Guadalajara

Sarah Pink

RMTT-Melbourne

Encartes, año 2, núm 3, marzo-agosto 2019, es una revista académica digital de acceso libre y publicación semestral editada por el Centro de Investigaciones y Estudios Superiores en Antropología Social, calle Juárez, núm. 87, Col. Tlalpan, C. P. 14000, México, D. F., Apdo. Postal 22-048, Tel. 548735 70, Fax 56555576. Directora de la revista: Ángela Renée de la Torre Castellanos. Alojada en la dirección electrónica https:// www.encartesantropologicos.mx. ISSN: 2594-2999. Las opiniones expresadas por los autores no necesariamente reflejan la postura de la revista. Se autoriza la reproducción parcial de los materiales publicados siempre y cuando se haga con fines estrictamente no comerciales y se cite la fuente. Salvo excepciones explicitadas, todo el contenido de la publicación está bajo una Licencia Creative Commons Atribución-NoComercial 4.0 Internacional. 